XVIII.

\title{
Die psychiatrische und Nervenklinik zu Königsberg.
}

\author{
Von \\ E. Meyer. \\ (Hierzu Tafeln XXX-XXXII und 6 Textfiguren.)
}

Im Juli 1913 ist die psychiatrische und Nervenklinik zu Königsberg eröffnet, als letzte gleichartiger Kliniken an den preussischen Universitäten.

Dem Namen nach bestand in Königsberg schon eine Reihe von Jahren eine psychiatrische Klinik; und zwar seit 1903 in der Weise, dass die psychiatrische Abteilung der städtischen Krankenanstalt von der Stadt dem Staat für den Unterricht überlassen war, mit einem Direktor in der Person des Vertreters der Psychiatrie an der Universität.

Von einer Klinik im wahren Sinne des Wortes, die sich mit denen an den anderen preussischen oder deutschen Universitäten vergleichen konnte, war aber nicht die Rede.

Ich möchte davon absehen, bei den unerfreulichen Bildern der Vergangenheit zu verweilen, nur sei so viel gésagt, dass die verfügbaren Räume trotz aller Aufwendungen gleich ungeeignet zur Behandlung und Beobachtung der Kranken, wie für Unterricht und Forschung blieben. Dass diese Einrichtung somit nur ein Notbehelf sein konnte, der möglichst schnell durch eine moderne Klinik ersetzt werden musste, konnte nicht geleugnet werden und wurde auch von der Staatsregierung, insbesondere dem Kultusministerium, sehr bald anerkannt. Wenn trotzdem erst 1913 die neue Klinik eröffnet werden konnte, so lag das an vielerlei Schwierigkeiten, u. a. den Baubeschränkungen im Festungsgelände, deren Ueberwindung viel Zeit erforderte.

Nicht einfach war die Platzfrage zu lösen.

Auf der einen Seite liessen Unterricht wie Forschung möglichst nahe Nachbarschaft mit den anderen Kliniken und Instituten geboten erscheinen, ebenso wie das Interesse der Kranken. Es war ja der einzige, aber nicht geringe Vorzug der alten Einrichtung, dass die psychiatrische Klinik ein Teil eines allgemeinen Krankenhauses war, und es lag die 
Befürchtung nahe, dass durch eine abgesonderte Lage die Voreingenommenheit und das mangelnde Verständnis des Publikums gegenüber psychisch Kranken neue Nahrung bekommen würde.

Andererseits war im Bereich der anderen Kliniken und ihrer näheren Umgebung, jedenfalls zu jener Zeit, kein Platz von annehmbarer Preislage und ausreichender Grösse - selbst bei Verzicht auf grössere Gartenenlagen - vorhanden. Es kam auch binzu, dass die klinischen wie die anderen Institute zum Teil eines Neubaues dringend bedüritig waren, und der Gedanke, sie alle aus der Innenstadt heraus zu verlegen, viel. fach erörtert wurde. Dazu kam, dass die Entfestigung der Stadt vor der Tür stand und dass mit Bestimmtheit zu erwarten war - wie es auch eingetreten ist - dass das Gebiet ausserhalb der Wälle sehr schnell mit privaten und öffentlicben Gebäuden bebaut werden würde, und immer bessere Verbindung mit den älteren Stadteilen und somit auch den innerhalb der Wälle liegenden Kliniken und Instituten erhalten würde.

Alles das liess die Bedenken gegen das jetzt gewählte, ausserhalb der Wälle liegende Terrain zurücktreten, selbst wenn die anderen Kliniken nicht ihren Platz wechseln würden.

Die Klinik hat etatsmässig eine Belegung von 80 Betten, ihre Gesamtkosten betrugen ca. $980000 \mathrm{M}$., das sei noch eingefügt.

Die Klinik liegt auf einer Art Anhöhe an der inneren Grenze eines ausgedehnten Villenviertels, von der Mehrzahl der anderen Kliniken und Institute zur Zeit höchstens 20-25 Minuten entfernt - in absehbarer Zeit werden neue Strassen eine wesentliche Verkürzung des Weges noch bringen -, wenige Minuten voll den Haltestellen mehrerer elektrischen Linien, die voraussichtlich auch noch näher kommen werden. Andererseits liegen parkartige Anlagen ihr vorgelagert, sodass ein Eingebautwerden dauernd verhindert ist. Sie befindet sich so gewissermassen auf dem Lande und doch in der Stadt.

Die Pläne der Klinik, die zuerst im Jahre 1907 entworfen wurden, Jehnen sich an die der psychiatrischen und Nervenklinik zu Breslau an. Wie dort, sind die eigentlichen klinischen Räumlichkeiten, Krankensäle, Auditorien, Laboratorien und dergleichen in einem Bau zusammengefasst, während das Wirtschaftsgebäude mit Kesselhaus and Beamtenwohnhaus sowie Leichenhalle und andererseits das Direktorwohnhaus getrennt liegen. Im Laufe der Zeit hat sich aus diesem ersten Entwurf der Grundriss herausentwickelt, den die Klinik jetzt aufweist (Taf. XXX).

Die Haupteinfahrtstrasse geht zwischen Direktorwohnhaus und Wirtschaftsgebäude im Bogen zu der Front des Hauptgebäudes; Wirtschaftsgebäude, Kesselhaus und Leichenhalle haben eine eigene Zufahrt. Während in Breslau das Wirtschaftsgebäude zwischen den Flügeln des 
Hauptgebäudes eingelagert ist, war das hier, da zwischen den Gängen, die das Hauptgebände mit den Pavillons verbinden, nur ein weit geringerer Abstand ist, nicht angängig, sodass die Verlagerung an die Strassenfront gewählt wurde. Der Nachteil, den in allen Fällen die Lage von Waschküche nnd speziell Küche ausserhalb des die Krankensäle enthaltenden Gebäudes hat, macht sich durch die damit gegebene grössere Entfernung und das Fehlen unter- oder oberirdischer Verbindungsgänge bei dem Transport der Speisen usw. besouders fühlbar. Andererseits ist die völlige Absonderung des ganzen wirtschaftlichen Betriebes von grossem Wert und ist meines Erachtens der Einrichtung der Küche usw. in Krankengebäude selbst vorzuziehen.

Auf die Einzelheiten der Einrichtungen des Wirtschaftsgebäudes (Fig. 2) gehe ich nicht ein; ich erwähne nur, dass die Klinik Warmwasserheizung hat, die von dem Kesselhaus ausgeht, wo auch die Warmwasserbereitung stattfindet.

Das Hauptgebäude (Fig. 1) besteht aus dem - völlig über der Erde gelegenen - Untergeschoss, dem Hauptgeschoss, dem Ober- und dem ausgebauten Dachgeschoss. Die Verteilung ist die gewöhnliche: Im gesamten Mittelbau und dem Dachgeschoss liegen die Poliklinik und die anderen therapeutischen Räume, das Auditorium mit dem Zimmer des Direktors und dem Vorzimmer, die Laboratorien und $\mathrm{Zu}$ behör, die Wohnungen der Aerzte und Verwaltung u. dergl., die Flügel nehmen die Krankensäle ein (Taf. XXXI u. XXXII).

Der Haupteingang führt, die Pförtnerloge rechts lassend, geradeaus in das Untergeschoss, während andererseits, von dem Haupteingang ausgehend, die Haupttreppe bis zum Obergeschoss hinauffübrt und auf dem Korridor vor dem Auditorium mündet. Sie findet ibre Fortsetzung in einer schmaleren Treppe, die den Aufgang zum Auditorium für die Studierenden bildet und bis in das Dachgeschoss weitergeführt ist. Vom Untergeschoss geht ausserdem vor den Krankenabteilungen jederseits eine Treppe ebenfalls bis zum Dachgeschoss hinauf. Alle Treppen und Korridore haben Linoleumbelag auf Korkelit, nur das Untergeschoss hat im Korridor Fliesenbelag. Betreten wir von dem Haupteingang aus das Untergeschoss, so findet sich in der Mitte ein grosser Raum für therapeutische Uebungen, daneben auf der einen Seite die therapeutischen Bäder mit Doucheapparat, elektrischem Wannenbad, Vierzellenbad, Kohlensäurebad, elektrischem Lichtbad usw., gegenüber das Aerztekasino. Auf der anderen Seite liegen nach vorn und binten das chemische bzw. das serologische Laboratorium. In den beiden Flügeln sind die Zimmer und der Aufenthaltsraum für die Pflegerinnen auf der einen Seite, auf der anderen die für die Pfleger, Heizer, Hausdiener usw. mit 
zugehörigem Bad und Klosett. Den Fussboden bilden in den bewohnten Räumen Stabfussboden, in den therapeutischen Bädern Lattenbelag auf Zementestrich, in den Laboratorien Flieseu.

Die Haupttreppe mündet im Hauptgeschoss (Taf. XXXI u. Fig. 5) gerade gegenüber der Poliklinik, die jederseits einen Warteraum, Abfertigungsraum mit Dunkelzimmer und ein Zimmer für besondere Unter-

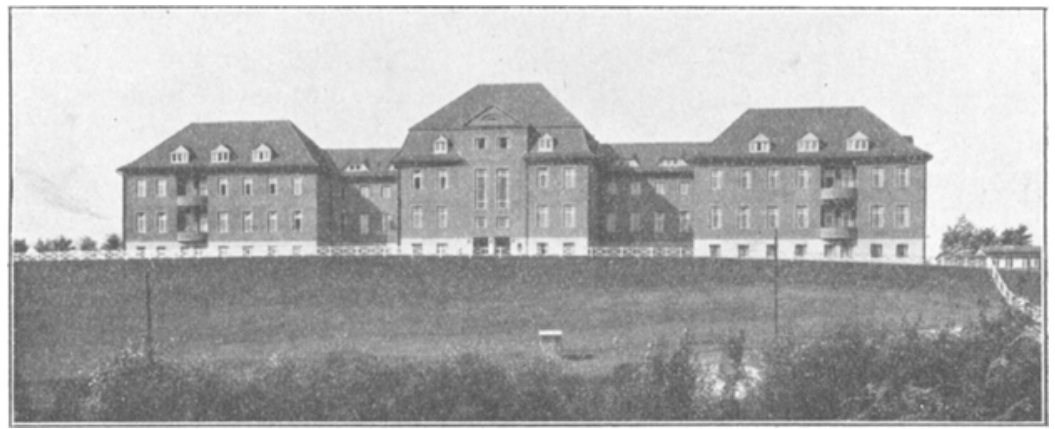

Fig. 1.

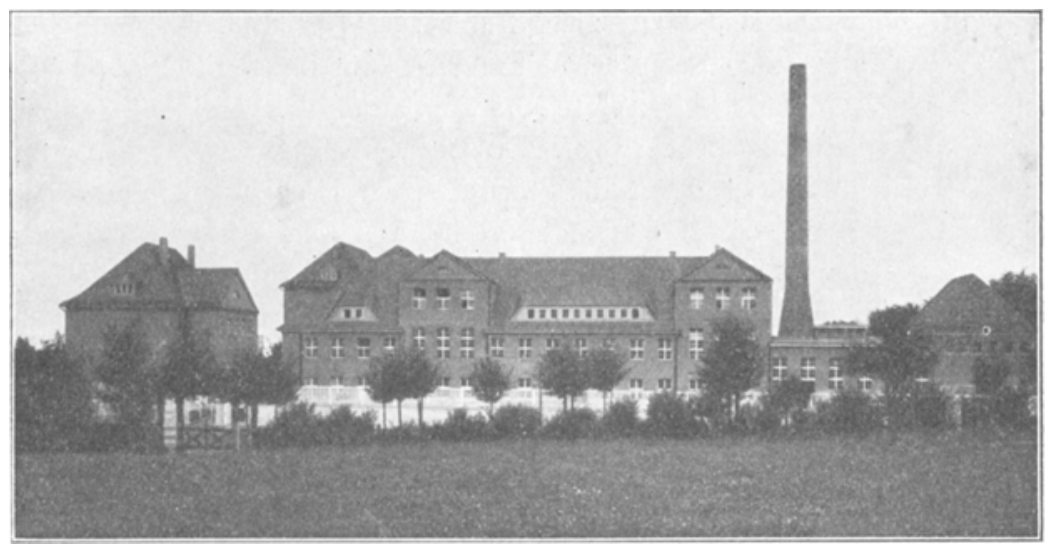

Fig. 2.

suchungen hat, mit elektrischen Apparaten verschiedener Art usw. ausgestattet ist. Gegenüber liegen auf der einen Seite zwei Zimmer für einen Assistenzarzt, auf der auderen das Aufnahmezimmer und die Verwaltung. Alle diese Räume haben ebenfalls Linoleumbelag auf Korkelit. Jederseits führen uns Glastüren in die Aufnahmeabteilungen. In diesen liegen (Taf. XXXI) an dem breiten Korridor, der geradeaus in den Wachsaal (8 Betten-Raum) führt, rechts das ärztliche Untorsuchungszimmer, die 
Nebenräume und ein Pflegezimmer sowie ein Dauerbad mit zwei Wannen, dessen Einrichtung ich weiterhin besprechen werde. Linkerhand folgt auf ein Einzelzimmer für ein oder zwei Kranke ein Fünfbettenraum und der Tagesraum mit kleinem Balkon. Der nun folgende Achtbettenraum, der nach der anderen Seite wieder an das Dauerbad grenzt, mit dem er durch eine Tür verbunden ist, bat nach Osten und Westen je zwei Fenster; die Betten sind so angeordnet, dass sie von rechts und links Licht erhalten. - Auf die wichtigsten Punkte der Einrichtung dieses Saales komme ich zurück.

In diese Aufnahmeabteilung kommen sämtliche Kranken mit psycbotischen Erscheinungen, abgesehen vou sehr unruhigen, die gleich in den Pavillon verlegt werden.

Nach letzterem führt ein $30 \mathrm{~m}$ langer, mit Linoleum ausgelegter und heizbarer, $1,75 \mathrm{~m}$ breiter Gang, der gleich hinter der Tür zur Aufnabmeabteilung, von der ihn eine besondere Tür trennt, abgeht.

Die Tür vom Gange in den Pavillon öffnet sich auf einen geräumigen Korridor, an dem die verschiedenen Nebenräume liegen. Vom Korridor treten wir in einen Saal für sechs Kranke, an den sich auf der einen Seite ein Dauerbad für zwei Wannen, auf der anderen ein Saal für vier Kranke anschliesst, der in einen Tagesraum mündet, der wieder mit dem Korridor verbunden ist (Taf. XXXI).

Steigen wir zum Obergeschoss hinauf, so bildet hier die Mitte das Auditorium, das für ca. 70 Zuhörer eingerichtet ist, mit ansteigendem Gestühl. Es besitzt eine Verdunkelungsvorrichtung mit Handbetrieb. In der Mitte des Zuhörerraums ist ein Leitz'sches Epidiaskop aufgestellt, für das die Wand hinter der Tafel als Projektionsfläche dient. Das Zimmer des Direktors mit Vorzimmer, in welchem die Kranken für die Vorstellung in der Klinik usw. warten können, schliesst sich an das Auditorium an. Die übrigen Teile des Mittelbaues im Obergeschoss nehmen zwei grosse und ein kleineres, für den Direktor bestimmtes, Laboratorium für mikroskopische und anatomische Arbeiten, sowie zwei kleine Zimmer für psychologische Untersuchungen ein. Beide Flügel bilden die Nervenabteilungen, die in ihrer Anordnung der darunter befindlichen Aufnahmeabteilung in dem für Kranke dritter Klasse bestimmten Achtbettenraum, dem Tagesraum, sowie - im wesentlichen - in den Nebenräumen entsprechen (Taf. XXXII). Der übrigbleibende Raum ist in jederseits sechs Zimmer mit 1 bzw. 2 Betten für Kranke erster und zweiter Klasse aufgeteilt.

Das Dachgeschoss endlich enthält in der Mitte die Wohnungen für die Assistenz- und Volontärärzte mit Ausnahme der erwähnten zwei Zimmer im Hauptgeschoss, samt Zubebör, ferner die Bibliothek, die 
anatomische Sammlung, das photographische Zimmer, in dem auch der mikrophotographische Apparat aufgestellt ist. In den Seitenteilen befinden sich u. a. die Wohnung der Oberin, die Zimmer für die Hausmädchen, die Räume für Aufbewahrung der Krankenkleider.

Damit ist die Aufzählung der hauptsächlichsten Räume der Klinik erledigt, die ich, schon weil ein Abdruck der Pläne aller Geschosse untunlich erschien, nicht unterlassen zu dürfen glaubte, so wenig anschaulich sie an sich sein kann.

Einer besonderen Besprechung bedürfen einmal die Krankensäle (Fig. 6). Ihr Fussboden wird ebenfalls von Linoleum, wie überall von grüner Farbe, auf Korkelit gebildet, die Betten sind aus Eisen, von dem gewohnten Typus moderner Krankenbausbettstellen, mit dreiteiligen Matratzen, die einen waschbaren Ueberzug haben. Sie sind weiss gestrichen, ebenso die Türen, Fenster, Schränke, Tische und Stühle, nur zum Teil farbig abgesetzt. Die Tische haben Lederstoffüberzug.

Ein Wort noch über die Fenster! Es sind Doppelfenster, die dreiteilig sind, dazu ein oberes Kippfenster. Das Mittelstück bleibt für gewöhnlich geschlossen und hat einen anderen Dornverschluss als die schmalen, etwa $9 \mathrm{~cm}$ weit zu öffnenden Seitenteile, die wie die Kippfenster zum Lüften dienen. Besondere Entlüftungseinrichtungen bestehen nicht.

Neben zwej Waschbecken aus Steingut mit Dornverschluss zum Ein- und Ablassen des Wassers ist ein kieines Becken zum Mlundausspülen, mit Wasserauslass darïber, angebracht. Ueber der Wascheinrichtung finden sich kleine Schränkchen aus Holz, seitlich mit Gittern zum Lüften versehen, zur Aufnahme von Wasserglas, Kamm und Zahnbürste für jeden Kranken, die ausserdem Spiegel an der Innenseite ihrer Türen haben. Feststehende Klosetts im Krankensaale babe ich vermieden, unter anderem wegen des Geräusches der Wasserspülung. Es sind dafür in allen Wachsälen und in den Dauerbädern transportable Nachtstühle aus weissem emailliertem Eisenblech im Gebrauch, von einer Wellwand verdeckt.

Die Krankensäle haben Deckenbeleuchtung aus mattem Glas, für die Nachtbeleuchtung ist in der mittleren Kuppel eine 5- bzw. 10kerzige Kohlenfadenlampe mit besonderer Schaltung angeordnet. Ausserdem ist eine Ansteckdose für elektriscbe Apparate und für den Kochapparat für die Nachtwache vorhanden. Zu erwähnen ist noch die Haustelephonstelle (in einem Wandschrank in der Wand) und Klingelleitung zum Pflegerzimmer auf der gleichen Abteilung. Die Decken sind weiss, ebenso die Wände im oberen Teil, während der untere (2 $\mathrm{m}$ hoch) mattfarbigen Oelfarbenanstrich erbalten hat. 
In sämtlichen Sälen sind bunte Leinenvorhänge zum Werfen, in den Pavillons nur Lambrequins, die Vorhänge ersetzen dort Jalousien.

Das eben entworfene Bild gilt für alle Krankensäle der Aufnahmestation und des Pavillons, also für alle Abteilungen, die für ausgesprochene Psychosen bestimmt sind. Andere Massnahmen zur Sicherung wie die erwähnten an den Fenstern und dergl. erschienen nicht nötig, nur ist noch zu erwähnen, dass die Türen nur durch einen Drücker zu öffnen und mit einem nach unten hakenförmig gebogenen Griff durch Zuziehen zu schliessen sind. Weiter haben die Lichtschalter besondere Schlüssel.

Dass in den Klosetts und auch überall sonst nach Möglichkeit alles ausgeschaltet ist, was Gefahr bringen könnte, bedarf keiner näheren Darlegung.

Die Nervenabteilung ist vollkommen offen, insbesondere werden die Türen nicht verschlossen gehalten. Wasser, Heizung und elektriscbes Licht sind jedem Kranken obne weiteres zugänglich, die Griffe an Türen und Fenster zeigen keine Besonderheiten. Nur sind in den Zimmern erster und zweiter Klasse an den Mittelfenstern Riegel mit einem Dornverschluss angebracht, um eine gewisse Sicherung im Notfalle zu ermöglichen.

Der in dem ursprünglichen Plan auf jeder Seite vorgesebene Isolierraum ist fortgelassen und in ein Zimmer für Kranke erster und zweiter Klasse bzw. für körperlich Schwerkranke umgewandelt. Ich erwähne das deshalb, weil Heilbronner bei der Beschreibung der Utrechter Klinik kürzlich die Ansicht vertreten hat, dass Isolierräume nicht vollständig entbehrlich seien. Ich glaube, dass er sich damit im Gegensatz zu der überwiegenden Mehrzahl der Fachgenossen befindet. Meine eigenen Erfahrungen lassen auch Isolierräume in keiner Weise notwendig erscheinen. Mehrere Jahre haben wir in den denkbar engsten und ungünstigsten Verhältnissen mit etwa 500 Aufnahmen jährlich gearbeitet, wo auch Dauerbäder ganz unausführbar waren. Trotzdem haben wir nie die Möglichkeit auch nur erwogen, Isolierräume einzurichten. Und in der Zeit von mehreren Monaten, in denen die Klinik jetat besteht, hat sich das Bedürfnis danach in keinem Falle geltend gemacht, trotz der zum Teil ausserordentlich erregten Kranken, wie sie besonders das benachbarte Russland aus der littauischen Bevölkerung uns bringt.

Die Dauerbäder (Fig. 3), von denen jede Seite zwei zu je zwei Wannen besitzt, haben emaillierte gusseiserne Badewannen, die ich auch wegen dex erheblichen Kosten von Wannen anderer Arten gewählt habe und die sich bis jetzt gut bewähren. Die Batterien stammen von der Firma F. Butzke \& Co., Berlin S 42 und sind so eingerichtet (vergl. 
Figg. 3 und 4), dass zuerst mit der Füllbatterie die Wanne gefüllt wird. Dann wird diese abgestellt und die Zulaufbatterie, die den ständigen Zufluss besorgt (durch Oeffnungen am Boden der Wanne tritt das Wasser ein und läuft durch den Ueberlauf $a b$; ausserdem ist ein besonderer Ablauf für Ablassen des Wassers vorbanden), angestellt. Menge und Temperatur des nun dauernd zufliessenden Wassers lässt sich durch Ventileinstellung bzw. durch den Drehkopf (bei $\mathrm{T}$ in der Figur) so genau einstellen, dass höchstens kleine Korrekturen weiterhin nötig sind. Die ganze Batterie befindet sich in einem eisernen Schutzkasten, der verglaste Ausschnitte für das Ablesen des Thermometers und das Einsetzen der Dreikante hat.

Die Laboratorien sind von mir schon namhaft gemacht. Wir verfügen, um es noch einmal hervorzuheben, über zwei bzw. drei anatomische bzw. mikroskopische Laboratorien, ein psychologisches, ans zwei Zimmern bestehend, je ein chemisches und ein serologisches Laboratorium, dazu kommen noch die anatomische Sammlung und das photographische Zimmer. Zum Teil sind diese Arbeitsräume schon völlig eingerichtet, zum Teil sollen sie in absebbarer Zeit ihre Ausrüstung erhalten, so dass nach allen Richtungen hin Untersuchungen ermöglicht sind.

Ein Stall für Versuchstiere und ein Operationsraum für Tierexperimente sind Erfordernisse, die baldmöglichst befriedigt werden müssen.

Die architektonische und dekorative Ausgestaltung der Klinik näher zu besprechen, kann nicht meine Aufgabe sein. Nur so viel sei gesagt, dass überall das Streben nach Licht und Farbe bervortritt (vgl. Figg. 5 und 6). Dafür sorgen die Glastüren am Eingang der Abteilungen wie an allen Nebenräumen und den Tagesräumen, das zeigen die verschiedenartigen Anstriche in Korridoren, Krankensälen, Einzelzimmern, Bädern usw., die bunten Vorhänge, die farbig abgesetzten Möbel usw.

Von allgemeinen Einrichtungen bebe ich hervor die Fernwarmwasserheizung und Warmwasserbereitung, die warmes Wasser neben kaltem ausser in die Säle und Zimmer der Patienten, in die Wohnungen der Aerzte, die ärztlichen Untersuchungszimmer, die Laboratorien liefert. Die Beleuchtung ist überall elektrisch, Gas finden wir in den Anrichten, dem Kasino, den Laboratorien.

Zur Erleichterung des Verkehrs dient ein Personenaufang, ferner ein Haustelephon mit Linienwählersystem und Zentrale. In den Haupträumen finden sich elektrisch betriebene Uhren.

Die Gärten sind mit niedrigem Drahtzaun versehen, nur die der Pavillons mit $2 \mathrm{~m}$ boher Planke, mit Rücksicht auf die unruhigen Kranken. 
Die psychiatrische und Nerrenklinik zu Königsberg.

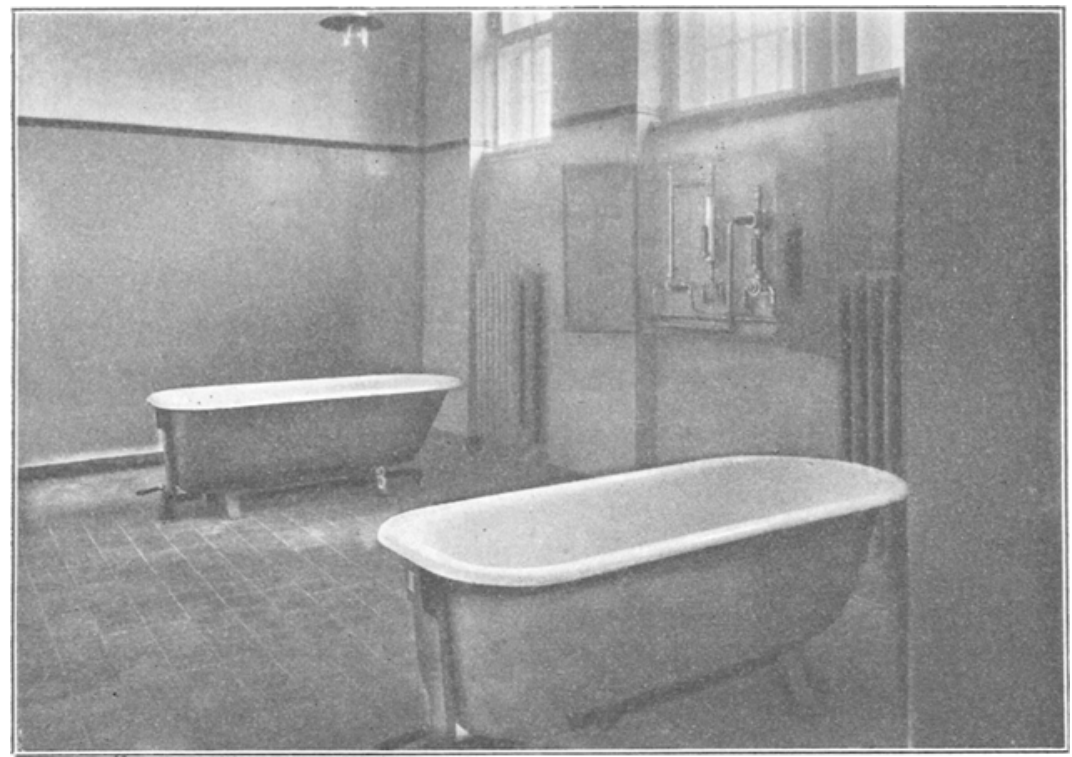

Fig. 3.

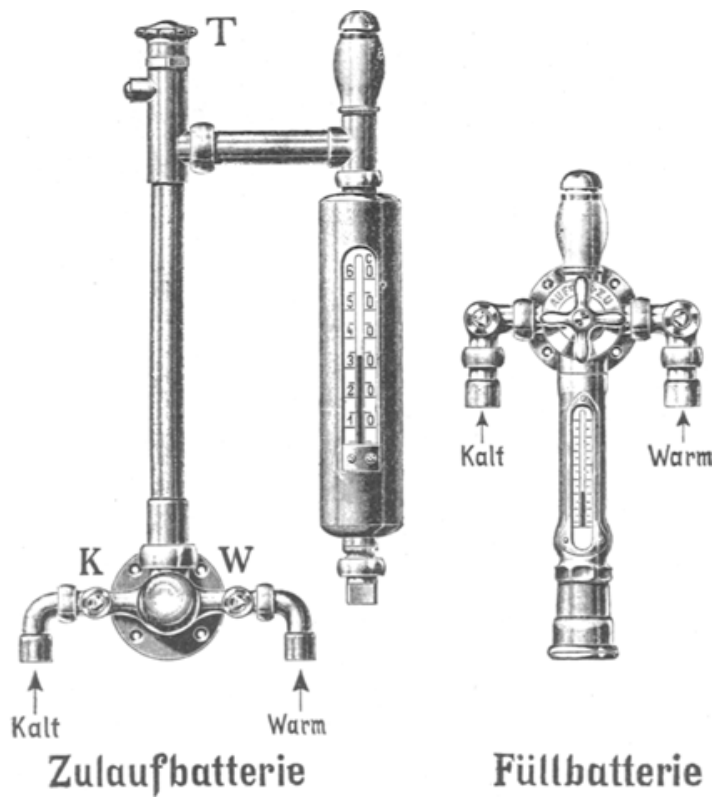

Fig. 4. 
Der Wachdienst wird von Dauerwachen wahrgenommen, die für 3-4 Wochen die Wache übernehmen, die Kontrolluhren sind Schreibwachuhren nach Greifswalder Muster (Fabrik von G. Hallwig, Stettin, Paradeplatz 3), die sich sehr gut bewähren.

Als Personal habe ich mich bemüht, Schwestern zu bekommen, doch ist mir das nicht gelungen. Ich bin daher auf Pfleger und Pflegerinnen unter der Leitung eines Oberpflegers und einer Oberin an gewiesen gewesen und bin bisher damit gut gefahren. Insbesondere hat sich das männliche Pflegepersonal, das ja erfahrungsgemäss gerade in grösseren Städten wenig zuverlässig erscheint und oft wechselt, wider Erwarten sehr gut bewährt.

Die 80 Betten, die der Etat für die Klinik vorsieht, sind so verteilt, dass jederseits die Aufnahmeabteilung 14, der Pavillon 10 enthält und der Rest von 16 der Nervenabteiling zufällt. Die Bettenzahl im Pavillon hatte ich absichtlich gering angesetzt, die unerwartete Hellhörigkeit des Hauses machte es aber nötig, den Pavillon stärker zu belegen, und brachte es so leider auch mit sich, dass die Dauerbäder in der Aufnahmeabteilung nicht voll ausgenützt werden können.

Das Bedenken, ob nicht die Lage der Klinik einer guten Belegung hinderlich sein würde, hat sich als völlig unbegründet erwiesen, wie die Aufnahmeziffern aus den ersten 4 Monaten zeigen mögen: Es wurden nämlich aufgenommen im. Juli 82, im August 78, im September 86 und im Oktober 77 Kranke, also im Monat durchschnittlich ebensoviel, wie überhaupt Betten zur Verfügung stehen.

Bei den Besichtigungen der Klinik ist 1 . a. die Frage an mich gerichtet, ob ich jetzt nach Abschluss des Baues und der Einrichtung wesentliche Ausstellungen hätte. Das glaube ich verneinen zu können. Selbstverständlich ergeben sich im klinischen wie Wirtschaftsbetriebe Yängel, die den Wunsch nach Abhilfe und Verbesserungen wecken. So zeigt sich, dass Betoneisenbau, wie ihn die Rlinik erbalten hat, trotz des dämpfenden Linoleumbelages auf Korkelit das Haus recht geräuschvoll und das Störende der Leitungen usw. besonders fühlbar macht.

Das Fehlen eines gedeckten Ganges oder dergl. zwischen Hauptgebäude und Küche ist auch hier zu nennen, ebenso das von verglasten Veranden und Rampen nach den Gärten. Auch machte sich schon sehr bald der Mangel eines eigenen Hauses für die Nervenkranken und die Kranken I. und II. Klasse hemerkbar.

Nabe liegt auch der Gedanke, die sämtlichen Arbeitsräume in einem vielleicht mehr gesonderten Teil der Klinik zu vereinigen, doch war das bei dem gegebenen Umfange nicht zu erreichen. 
Die psychiatrische und Nervenklinil zu Königsberg.

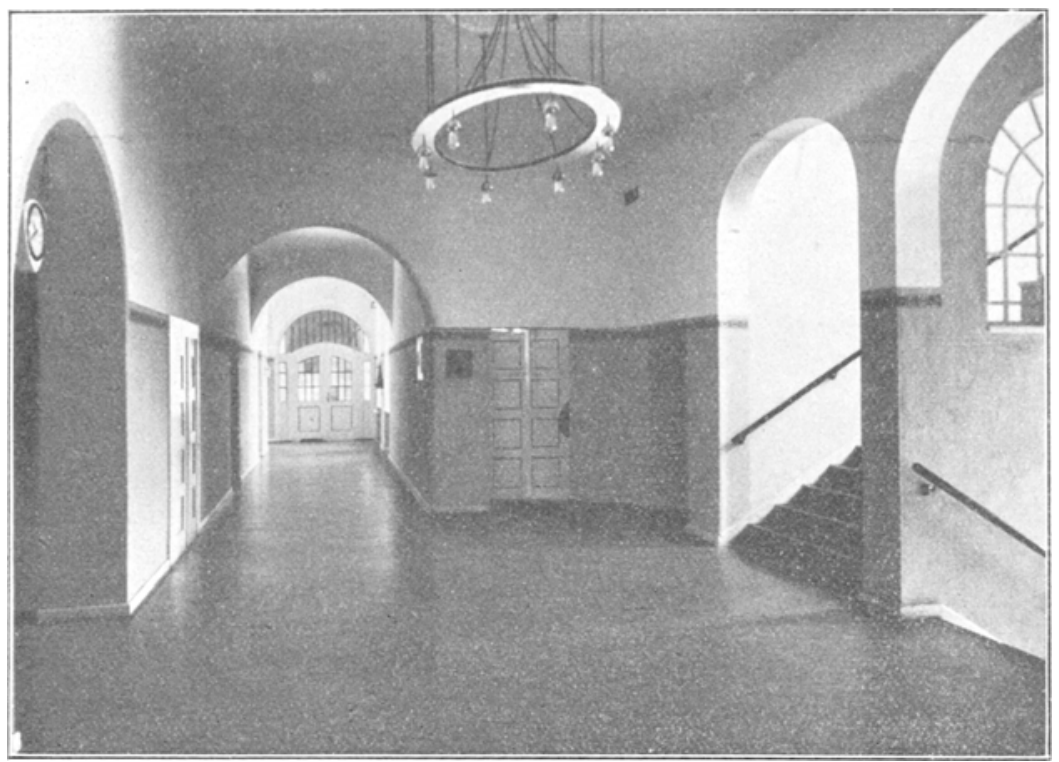

Fig. 5.

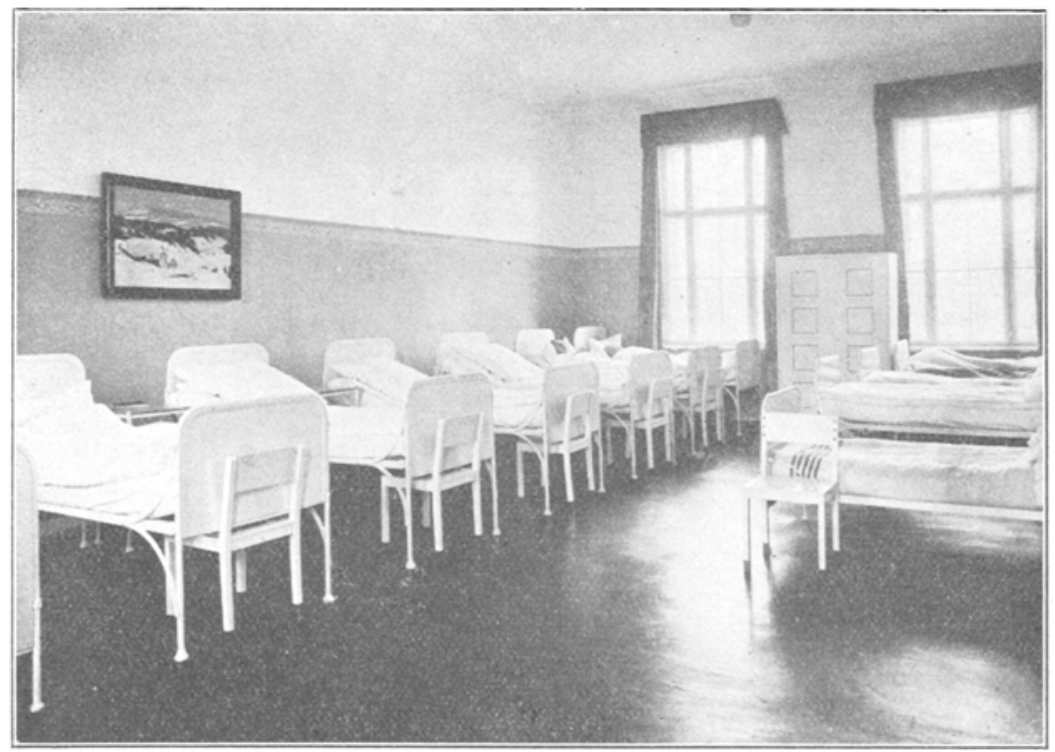

Fig. 6. 
684 E. Meyer, Die psychiatrische und Nervenklinik zu Königsberg.

Mein Wunsch war es, die Sicherungsmassregeln, deren Zahl und Ausdehnung ja schon gering ist, noch mehr einzuschränken, um dem Ideal eines allgemeinen Krankenhauses immer mehr nahe zu kommen und so vor allem die Beobachtung und Behandlung möglichst günstig zu gestalten. Doch das wird sich nur mit besonders zahlreichem und und beständigem Pflegepersonal und sorgfältigster langdauernder Ausbildung verwirklichen lassen.

Dem Gesamteindruck gegenüber treten aber diese Ausstellungen weit zurück und wir werden alles in allem dankbar anerkennen, dass der Staat hier ein in jeder Weise für Unterricht, Behandlung und Forschung gleich geeignetes Institut geschaffen hat.

\author{
Erklärnng der Abbildungen (Tafeln XXX-XXXII). \\ Tafel XXX: Lageplan. \\ Tafel XXXI: Hauptgeschoss. \\ Tafel XXXII: Obergeschoss.
}

